\title{
QUEEN'S
UNIVERSITY
BELFAST
}

\section{Surface-Enhanced Raman Spectroscopy as a Probe of the Surface Chemistry of Nanostructured Materials}

Dick, S., Konrad, M. P., Lee, W. W. Y., Mccabe, H., Mccracken, J. N., Rahman, T. M. D., Stewart, A., Xu, Y., \& Bell, S. E. J. (2016). Surface-Enhanced Raman Spectroscopy as a Probe of the Surface Chemistry of Nanostructured Materials. Advanced Materials, 28(27), 5705-5711. https://doi.org/10.1002/adma.201505355

Published in:

Advanced Materials

Document Version:

Peer reviewed version

Queen's University Belfast - Research Portal:

Link to publication record in Queen's University Belfast Research Portal

\section{Publisher rights}

(c) 2016 Wiley-VCH Verlag GmbH\& Co, Weinheim.

This is the peer reviewed version of the following article: Dick, S., Konrad, M. P., Lee, W. W. Y., McCabe, H., McCracken, J. N., Rahman, T. M. D., Stewart, A., Xu, Y. and Bell, S. E. J. (2016), Surface-Enhanced Raman Spectroscopy as a Probe of the Surface Chemistry of Nanostructured Materials. Adv. Mater.., which has been published in final form at http://doi.org/10.1002/adma.201505355

This article may be used for non-commercial purposes in accordance with Wiley Terms and Conditions for Self-Archiving.

\section{General rights}

Copyright for the publications made accessible via the Queen's University Belfast Research Portal is retained by the author(s) and / or other copyright owners and it is a condition of accessing these publications that users recognise and abide by the legal requirements associated with these rights.

\section{Take down policy}

The Research Portal is Queen's institutional repository that provides access to Queen's research output. Every effort has been made to ensure that content in the Research Portal does not infringe any person's rights, or applicable UK laws. If you discover content in the Research Portal that you believe breaches copyright or violates any law, please contact openaccess@qub.ac.uk. 


\section{WILEY-VCH}

DOI: 10.1002/ ((please add manuscript number))

\section{Article type: Research News}

\section{Surface Enhanced Raman Spectroscopy as a Probe of the Surface Chemistry of Nanostructured Materials}

Susan Dick, Magda P. Konrad, Wendy W. Y. Lee, Hannah McCabe, John N. McCracken, Taifur Rahman, Alan Stewart, Yikai Xu, Steven E. J. Bell*

S. Dick, Dr M. P. Konrad, Dr W. W. Y. Lee, H. McCabe, J. N. McCracken, Dr T. Rahman, Dr A. Stewart, Y. Xu, Prof. S.E.J. Bell

School of Chemistry and Chemical Engineering, Queen's University, Belfast BT9 5AG, U.K. E-mail:s.bell@qub.ac.uk

Keywords: SERS, nanomaterial, sensor, SAM

Surface-enhanced Raman spectroscopy (SERS) is now widely used as a rapid and inexpensive tool for chemical/biochemical analysis. The method can give enormous increases in the intensity of the Raman signals of low concentration molecular targets if they are adsorbed on suitable enhancing substrates, which are typically composed of nanostructured Ag or Au. However, the features of SERS which allow it to be used as a chemical sensor also mean that it can be used as a powerful probe of the surface chemistry of any nanostructured materials which can provide SERS enhancements. This is important because it is the surface chemistry that controls how these materials interact with their local environment and in real applications this interaction can be more important than more commonly measured properties such as morphology or plasmonic absorption. Here the opportunity which this approach to SERS provides is illustrated with examples where the surface chemistry is both characterized and controlled in order to create functional nanomaterials.

\section{Introduction}

In the 40 years since its discovery surface-enhanced Raman spectroscopy (SERS) has evolved from a niche technique, studied by small number of specialist groups, into a mainstream 


\section{WILEY-VCH}

spectroscopic method. The advantages of SERS for detection, identification and quantification of molecular targets are now well known, in particular the huge enhancement factors which it can provide. ${ }^{[1]}$ Currently, the vast majority of SERS studies involve detection of target molecules which give SERS signals when they adsorb onto an enhancing surface. ${ }^{[2]}$ The sensitivity of SERS is such that it is appropriate for even sub-monolayer surface coverage, indeed in analytical applications the SERS response typically plateaus at higher concentrations when all the surface binding sites are occupied. ${ }^{[3]}$ While signalling the binding of extrinsic compounds is very useful for analytical/diagnostic applications and is the predominant application of SERS, the features of SERS which make it attractive as a chemical sensor also mean that it can be a valuable probe of the enhancing nanostructured materials themselves. For example, it can be used to study the surface chemistry of the asprepared materials, monitor subsequent modification steps or understand chemical reactions which occur on the surface. It is these aspects of SERS (illustrated in Figure 1), rather than conventional small molecule detection, which are discussed here.

\section{Characterization of SERS-active Materials}

SERS enhancing materials are typically composed of nanostructured coinage metals (overwhelmingly $\mathrm{Au}$ or $\mathrm{Ag}$ ) and basically divide into 2 main classes, those which have regular structure and those where the nanostructure is random. ${ }^{[1-3]}$ Examples of regularly structured materials include lithographically prepared substrates, such as Klarite ${ }^{[4]}$ and those prepared on templates ${ }^{[5]}$, while randomly structured substrates include nanoparticle aggregates $^{[1-3]}$ and roughened electrodes ${ }^{[1]}$. However, the number of materials which show the enhanced Raman scattering is now very large and growing rapidly. Moreover, materials intended for other uses may be SERS active, for example, electrolessly deposited fractal Ag on $\mathrm{Cu},{ }^{[6]}$ which is also used to create superhydrophobic surfaces. ${ }^{[7]}$ 


\section{WILEY-VCH}

There are already numerous tools available for characterising nanostructured materials and these are generally applicable to SERS-enhancing materials. For example, electron microscopy, STM and AFM can be used to determine morphology and dynamic light scattering, particle tracking and size exclusion chromatography for particle sizing. ${ }^{[8]}$ Similarly, the chemical composition of nanoscale and microscale objects can be inferred from elemental analysis by EDX and their plasmonic properties measured using optical spectroscopy. ${ }^{[8]}$ However, in nanomaterials which are required to interact with their environment it is the surface properties which are important and in the case of nanostructured metals these properties are often determined by a thin molecular layer on the material's surface whose composition is not readily studied by any of the above techniques. Of course it is often straightforward to measure the physical properties which the molecular layers provide, such as the contact angles of solid materials, but the options for direct investigation of their chemical composition and properties are much more limited. Ellipsometry is useful but provides limited chemical information and is limited to bulk samples. High vacuum techniques such as XPS can give information under stringently controlled conditions but are not appropriate for materials which are in solution.

In contrast to all of the above, SERS can be used to study either wet or dry samples in the open laboratory or as components within electrochemical cells. The measurements are typically rapid and can be carried out at micron lateral resolution using widely available microscope-based systems or at $\mathrm{nm}$ resolution using more specialised tip-enhanced approaches. ${ }^{[9]}$ Moreover, the cost of making simple bulk (> $10 \mu \mathrm{m}$ laser probe spot size) SERS measurements has dramatically decreased in the past decade due to the commercial availability of low cost, compact Raman spectrometers which are primarily designed for field analysis of bulk materials, either for industrial quality assurance/control or in defence/law enforcement applications but are equally useful for recording SERS signals. 


\section{WILEY-VCH}

\section{Information Available from SERS}

The most basic information given by SERS is that the appearance of the characteristic vibrational spectrum of a compound in the SERS signal of a material immediately demonstrates that the compound is located within the enhancing region, which extends a few $\mathrm{nm}$ at most from the material's surface. ${ }^{[1]}$ However, the spectra can give much more information than the simple presence of a particular molecule or ion on the surface, they also give information on the structure of the adsorbed molecules or ions. For example, the most widely studied molecular layers on noble metal surfaces are the self-assembled monolayers (SAMs) which spontaneously form when $\omega$-terminated thiols are allowed to react with Au (or less often Ag) surfaces. ${ }^{[10]}$ In this case SERS can be used to determine if the thiols are chemicaly attached to the surface or physisorbed, since chemisorption results in loss of S-H features, combined with the appearance of S-metal stretching bands. ${ }^{[1]}$ Moreover, if the surface layer is a long chain alkyl thiol the alkyl chain may have either trans or gauche conformation around the S-C bonds. Extensive studies have shown that the $v(\mathrm{C}-\mathrm{S})$ frequencies change significantly on gauche-trans reorientation with $v(\mathrm{C}-\mathrm{S})_{\mathrm{T}}$ at $c a .735 \mathrm{~cm}^{-1}$ and $v(\mathrm{C}-\mathrm{S})_{\mathrm{G}}$ at $c a .655 \mathrm{~cm}^{-1} .{ }^{[11]}$ Indeed this effect has been used to observe the evolution of adsorbed layers, from initially formed highly disordered systems to much more ordered layers. ${ }^{[12]}$

The final type of information that SERS can provide is orientation of the adsorbed molecules with respect to the metal surface. Due to the directionality of the plasmonic adsorption the selection rules for SERS are different from those of the same molecules in solution; the modes with polarizability changes perpendicular to the surface are preferentially enhanced and dominate the SERS spectrum. By determining the enhancement of appropriate modes the orientation of their polarizability tensors and therefore the associated, molecular framework can be deduced. ${ }^{[13]}$ Typically SERS will be used for exterior surface species but more recently it has also been used to probe the internal structure of $\mathrm{Au}$ nanostars 


\section{WILEY-VCH}

encapsulated or partly encapsulated within a Au outer shell. In these systems shifts in the band positions of 1,4-benzenedithiol on the nanostar core were attributed to changes in orientation caused by overgrowth of the outer Au shell. ${ }^{[14]}$

\section{Applications of SERS to Nanomaterial Characterisation}

\subsection{Characterisation of Sensor Surfaces}

An ongoing challenge within SERS research is understanding the factors which determine the performance of SERS sensing systems. In particular, it is important to understand the role which the sensor's surfaces, rather than their plasmonic properties, play in the detection of small molecule targets. Since enhancement only occurs for molecules at the surface, the chemical factors which promote or prevent target binding are clearly crucial to correct operation of the sensor. Under the experimental conditions used for practical analytical SERS measurements, the surfaces of the enhancing materials, which can be either metal nanoparticles or enhancing solid substrates, are typically not bare metal but instead carry a surface layer of some type. With evaporated films this will typically be metal oxide but with chemically reduced materials it can be one or more of the compounds added or created during the synthesis. ${ }^{[3]}$ Most obviously this could be the characteristic citrate layer which is observed on the surface of citrate-reduced silver colloid or the chloride layer found on freshly prepared hydroxylamine reduced silver but it could also be residual surfactant if the materials were prepared by template synthesis or electrolyte ions in electrochemically roughened electrodes. ${ }^{[1]}$ In addition, for aggregated particles the aggregating agent may itself adsorb to the surface. ${ }^{[15]}$ Even imaging nanoparticles under SEM has been shown to create an amorphous carbon layer which gives a characteristic SERS signal. ${ }^{[17]}$

It is important to understand the chemistry of these surfaces because in the analytical procedure it is these surfaces which the target interacts with, not the underlying metal. These substrate-target interactions are complex but a useful simplification is to separate the cases 


\section{WILEY-VCH}

where the analytes interact with the enhancing surface by co-adsorbing along with existing surface species from those where the existing species are displaced. Understanding which of these is appropriate for each combination of enhancing material and target allows rational design of materials which can be used as sensors for particular targets.

The simplest interaction is co-adsorption, for example, much of the early work on SERS used metal nanoparticles aggregated with simple alkaline halide salts (which created aggregates with negative $\zeta$ potentials) to detect positively charged analytes, which coadsorbed along with the surface halide. Conversely, anions are electrostatically repelled by such surfaces but can be detected by enhancing materials which are modified by $\omega$-terminated thiols such as $\mathrm{X}-\left(\mathrm{CH}_{2}\right)_{\mathrm{n}}-\mathrm{SH}$ where $\mathrm{X}$ is a quaternary ammonium salt, since these have a strongly positive zeta potential. ${ }^{[17]}$

A good example of the alternative displacement mechanism is in the detection of dipicolinic acid (DPA), which is a dicarboxylic acid which carries a +2 charge at neutral pH and is used as a proxy for anthrax spores. DPA can be detected using SERS with citratereduced colloid which has been aggregated with $\mathrm{MgSO}_{4}$ but not $\mathrm{NaCl}$, even though both materials have similar plasmonic properties. ${ }^{[18]}$ SERS spectra of the colloids aggregated with $\mathrm{MgSO}_{4}$ show that residual citrate ions from the preparation procedure remain in place after aggregation, since the sulfate ions do not displace them. In contrast, chloride aggregation causes the surface citrate to be replaced by chloride, as indicated by the appearance of a strong Ag-Cl band at $245 \mathrm{~cm}^{-1}$ and loss of the citrate bands. The successful detection of DPA with $\mathrm{MgSO}_{4}$ aggregation demonstrates that DPA can displace citrate from the surface while the failure with $\mathrm{NaCl}$ aggregated materials shows that the Ag-Cl bonds are too strong to allow DPA to replace the surface chloride. In fact, it is possible to carry out a sequential experiment where addition of DPA to $\mathrm{MgSO}_{4}$ aggregated colloid, followed by $\mathrm{NaCl}$ and then $\mathrm{KBr}$ causes the initial citrate spectrum to be replaced by DPA signals which are in turn replaced by Ag-Cl bands and then finally displaced by the formation of Ag-Br. ${ }^{[18]}$ 


\section{WILEY-VCH}

Similar effects are also observed with the DNA bases, which bind strongly to chloride modified Ag particles while the corresponding mononucleotides, which also carry negatively charged ribose sugar and phosphate groups do not bind, presumably because the additional electrostatic repulsion means they are unable to displace the existing chloride. ${ }^{[19]}$ In contrast, the mononucleotides are able to displace citrate and so can be detected using colloids which carry citrate on their surface.

These considerations make it obvious why it is important to monitor the nature of the surface chemistry of the sensors while they are in the actual working form. For example, aggregated silver colloids which are held within a dry swellable polymer (hydroxyethylcellulose) film have been prepared as off-the-shelf SERS sensors. ${ }^{[20]}$ Their principle of operation is that in the dry state the aggregates are protected within the host polymer but addition of aqueous analyte solution causes the films to swell, which releases the particles to interact with the target analytes and pulls the aqueous solution into the film to promote the interaction. In these films, citrate-reduced silver colloids are used in the preparation and aggregation is carried out using non-coordinating salts but in situ SERS measurements show that the active surfaces in the film carry an unintended surface chloride layer which is accidently created by ligand displacement during the film preparation step. This surface layer does not prevent detection of strongly binding test compounds or drugs of abuse but excess chloride does interfere with analysis of the therapeutic drugs which were the original target. ${ }^{[20,21]}$ Again, detecting the nature of the surface species on metal nanoparticles buried within a polymer host would be extremely challenging by any other analytical method but is extremely straightforward with SERS.

The final example of the utility of in situ SERS monitoring is in studies of Metal Liquid-Like Films (MeLLFs), which are mobile sheets of metal nanoparticles that sit at the interface between an aqueous and immiscible non-aqueous solvent. ${ }^{[22,23]}$ The films have remarkable properties, they typically have a highly reflective metallic appearance, despite 


\section{WILEY-VCH}

being just one nanoparticle layer thick, can self-heal if damaged and provide very strong SERS enhancements. They can be prepared by shaking an aqueous metal colloid with an immiscible non-aqueous solvent containing "modifier" compound which was originally believed to adsorb to the particles, increasing their hydrophobicity. This was supported by observation of strong SERS signals of the modifiers from the MeLLFs. ${ }^{[23]}$ However, recent studies have shown there are compounds which promote MeLLF formation without surface adsorption as shown by the SERS spectra of the MeLLFs, which are identical to those of the parent colloid. ${ }^{[24]}$ This SERS evidence was key in showing that there are two separate mechanisms for MeLLF formation based on increased particle hydrophobicity and/or decreased Coulombic repulsion between the particles.

\subsection{Monitoring Multicomponent Chemical Modification of Surfaces}

Surfaces which carry a layer of a single chemical compound are the simplest to prepare and/or characterize. The next step up in complexity is the preparation of materials carrying SAMs with mixed compositions, an approach which has been explored for many years and is currently experiencing something of a renaissance. Again, this area has been most thoroughly explored using mixtures of thiols as modifiers. In the simplest case, mixed thiol SAMs may be used for creating materials where only a small fraction of the surface carries the active modifying compounds, but the remainder of the surface needs to be passivated to prevent unwanted side effects. This is a very common practice in the preparation of functionalised nanoparticles intended for use in biological environment, for example, in passivation of particles conjugated with active compounds that function through targeted interactions such as antibodies or ss-DNA. ${ }^{[25]}$ In these systems non-specific binding, which gives large positive background signals, is minimised by passivating the inactive parts of the surface with a relatively inert layer of short chain alkyl thiols. Similarly, mixed charge monolayers have been used to improve the blood platelet compatability of gold surfaces. ${ }^{[26]}$ 


\section{WILEY-VCH}

A more ambitious approach is to create materials where the surface not only carries two or more modifiers but that these show cooperativity i.e. the combination of these modifiers creates areas on the surface which carry out a function which is different from that which can be achieved by either of the two component modifiers alone. For example, in studies of glucose detection by SERS, particle surfaces modified by a mixed decanethiol, $\mathrm{CH}_{3}\left(\mathrm{CH}_{2}\right)_{9} \mathrm{SH}$ and mercaptohexanol $\mathrm{HO}\left(\mathrm{CH}_{2}\right)_{6} \mathrm{SH}$ layer are particularly effective in promoting glucose absorption, since they are believed to create hydrogen-bonding hydrophobic "pockets” where the glucose can bind. ${ }^{[5]}$

Mixed surfaces can be prepared either by reacting with a single modifier and then carrying out place exchange reactions or by using a mixed modifier solution to carry out a single step modification. ${ }^{[10]}$ Of course, in the first case there is an obvious need for monitoring the surface composition during the exchange reaction and SERS is excellent for this purpose. However, even with the "one step" method there is a similar need to measure the surface composition because differences in the relative binding constants of the modifying compounds often mean that the relative proportions of the modifiers on the surface are different (sometimes to a large extent) from those used in the modifying mixture. For example, we have used SERS to study the compositions of mixed thiol modified metal nanoparticles. ${ }^{\text {[27] }}$ With these Au or Ag nanoparticles, the approach of using place exchange reactions is often prevented by the spontaneous formation of aggregates after the initial modification, which causes the particles to precipitate from solution before the subsequent place exchange reactions are complete. In contrast, the one step approach very rapidly creates the mixed composition surface monolayer which can then be characterised using SERS.

Since the relative SERS scattering cross-sections of each surface species can be determined, SERS measurements of relative peak heights give a simple and direct quantitative measurement of the relative proportions of each compound on the surface, as shown in Figure 2. The advantage of this approach is that it is sufficiently rapid to allow multiple 


\section{WILEY-VCH}

measurements to be made over a range of experimental conditions, so that the surface composition can be plotted against that of the modifier feedstock. If the binding of both compounds is identical then the mole fraction on the surface will match that of the bulk, as is observed with alkyl thiols with similar chain lengths. However, very dissimilar binding constants are expected, and found, for modifiers with different chemical compositions and this leads to examples where the surface composition is very far from that of the feedstock (see Figure 2d)). In addition, it has recently been found that the surface mole fraction depends not only on the relative binding constants of the components in the modifying feedstock, but also on the total concentration of modifiers. ${ }^{[27]}$ At first sight this observation is counterintuitive since the relative binding probability would be expected to be independent of the total concentration. However, this will only be true at high total concentration, where the loss of the modifiers from the feedstock due to adsorption makes a negligible difference to its composition throughout the process. At low concentrations this loss can have significant effects. The key parameter is the number of molecules of modifier available in the feedstock compared to the number of surface sites. If one of the thiols is much more strongly binding than the other, then the surface will be covered by it, even if the feedstock is an equimolar mixture of both modifiers. However, if the total number of molecules of modifiers of all types approaches the number of free sites then there is no competition for surface sites and the surface will have the same composition as the feedstock.

An example of a practical application of this approach is one where mixed SAMs composed of sodium 3-mercapto-1-propanesulfonate (MPS) and benzyl mercaptan (BZM) were used as materials for the enhanced binding of 3,4-methylenedioxymethamphetamine (MDMA). ${ }^{[28]}$ MDMA is the most widely used of the "ecstasy" family of drugs of abuse, which rose to prominence in the 1990's (associated with rave dance culture) and still continues to be a significant problem, despite the more recent rise of the newer generation of novel psychoactive substances. MDMA does not bind strongly to unmodified colloid surfaces, 


\section{WILEY-VCH}

nor does it bind strongly to surfaces which are modified exclusively by MPS or BZM. However, it does bind to surfaces prepared from mixed feedstocks, with the maximum binding at a feedstock composition of 60:40 MPS:BZM. This corresponds to a surface with approximately $31 \%$ MPS and $69 \%$ BZM, presumably this gives the best balance between aromaticity and negative charge to attract positively-charged MDMA.

This result demonstrates that there is cooperativity between the binding elements, which in turn suggests that the MDMA is able to interact simultaneously with both binding elements on the surface. This can only occur if there are at least some regions of the surface where the different thiols are in very close physical proximity. Conversely, if a mixed thiol particle is composed of large domains of each thiol, then the behaviour would be expected to be simply the weighted sum of the contributions from each domain. Recently tip-enhanced Raman imaging has been used to probe domain formation within binary self-assembled monlayers of oligomeric phenylene-ethynylene (OPE) thiol and thiophenol. These elegant experiments were used to determine that at various molar ratios of the coadsorbed thiols the surface layer phase segregated into domains $\sim 30-240 \mathrm{~nm}$. Moreover, it was also shown that reliable detection of these nanodomains required a pixel size less than $15 \mathrm{~nm} .{ }^{[29]}$ These experiments are clearly important since they provide a means of detecting domains based on chemical properties rather than the height differences used in AFM/STM, which is challenging on flat surfaces and has proved to be even more controversial for mixed thiol nanoparticles. ${ }^{[30]}$

\subsection{Monitoring Reactions on Surfaces using SERS}

The most obvious use of SERS for chemical reaction monitoring is to use it as a conventional analytical technique which can detect changes in the bulk concentration of reactants or products over time. However, within the current context it is useful to note that SERS also offers the opportunity to monitor reactions which occur directly on the surface. One of the 


\section{WILEY-VCH}

most thoroughly studied of these is the plasmon-assisted conversion of $p$-aminothiophenol to 4,4-dimercaptoazobenzene which was discovered by chance and was initially attributed to enhanced vibrations of the starting material under SERS probing rather than product formation. ${ }^{[31]}$ Conversely, chemical reactions of surface species to form characteristic products may be used for indirect detection of molecular targets, such as in the use of $\mathrm{Au}$ nanoparticles functionalised with 4-acetamidobenzenesulphony azide which forms the corresponding amide on reaction with $\mathrm{H}_{2} \mathrm{~S}$ and has been used to detect edogenous $\mathrm{H}_{2} \mathrm{~S}$ production in living cells. ${ }^{[32]}$

\section{3 (i) SERS Monitoring of Catalytic Surfaces}

A particularly promising application of SERS for surface reaction monitoring is in situ monitoring of catalysed reactions using multifunctional materials which have both catalytic activity and appropriate plasmonic properties. For example, Zhang et al et al have shown that multilayer materials composed of small $(15 \mathrm{~nm})$ catalytically active Au particles deposited on top of a self-assembled layer of larger $(56 \mathrm{~nm})$ SERS-active particles allows the catalysed reduction of $p$-nitrothiophenol to $p$-aminothiophenol by $\mathrm{NaBH}_{4}$ to be monitored. ${ }^{[33]}$ Similarly, SERS can be used as an in situ spectroelectrochemical probe of the active speces and products formed on electrode surfaces. ${ }^{[34]}$ In a more complex example, Ling et al prepared a layer of Ag nanocrystals protected by a thin alumina layer onto which was deposited a layer of $\mathrm{TiO}_{2}$ nanoplates decorated with Pt co-catalyst nanoparticles. ${ }^{[35]}$ Under UV irradiation peroxo, hydroperoxo and hydroxo water photo-oxidation intermediates were observed. In this work SERS was used throughout the preparation of the materials, initially to follow the removal of an interfering PVP layer on the Ag nanocubes and then to detect the overlayed $\mathrm{TiO}_{2}$ particles before finally recording spectra of the water-splitting products on the surface. These concepts have also been shown in free nanoparticles where $80 \mathrm{~nm}$ Au nanoparticles were protected with a thin inert silica shell prior to thiol functionalization and attachment of small 


\section{WILEY-VCH}

catalytically active Au nanoparticles which showed strong plasmonic coupling to the larger core. ${ }^{[36]}$ These particles were used to monitor the Au-catalyzed reduction of 4-nitrothiophenol, importantly it was found that the inert silica shell isolated the large Au core particle and thereby prevented it from interfering with the catalytic processes under investigation.

\section{3.(ii) Switching of surfaces using spiropyrans.}

Transformation of surface properties can also be achieved by physical rather than chemical stimuli. An excellent example is in the switching of photochromic spiropyrans on Au surfaces. Spiropyrans can exist as either the ring-closed spiropyran (SP) form or the ringopen merocyanine (MC) form. UV light is generally used to induce ring-opening of the SP to MC form, while the reverse process can be driven thermally or by irradiation with visible light. There has been considerable interest in preparing spiropyrans which can be used to create materials with optically switchable surfaces. One of the most successful studies was based on BIPS (3-(3',3'-dimethyl-6-nitrospiro[chromene-2,2'-indolin]-1'-yl)-ethyl-5-(1,2dithiolan-3-yl)pentanoate), a spiropyran with a long chain linker and a dithiolate surface anchor, shown in Figure $3^{[37]}$ This system can be thermally or optically (785 nm excitation) switched from the MC to the SP form and then reversed using UV excitation. Since the ring open and closed forms have different characteristic vibrational spectra, the chemical changes associated with the switching process can be monitored in situ using SERS at $785 \mathrm{~nm}$ so that changes in optical absorbance can be confirmed by SERS as arising through the intended mechanism. Surprisingly, in this study it was found that under the laser power densities used to record the SERS spectra, which were in the normal range for such measurements, the systems unexpectedly switched to the MC form, the opposite of what is expected under visible excitation. It was found that the power densities used were sufficient to cause twophoton switching of the SP-MC form and that the effect could be controlled by altering the excitation conditions so that it was possible to monitor the expected switching under low 


\section{WILEY-VCH}

power or induce switching in the opposite sense by increasing the irradiance of the Raman probe laser.

\section{Conclusion}

While the surface chemistry of nanomaterials is the aspect of their overall composition which controls their interactions with their surroundings, it is more difficult to characterise than the more commonly measured parameters such as morphology and plasmonic absorption. In part this is due to the low total concentration of surface species which will be present in the sample, but it is also because most methods for surface chemical analysis work best under high vacuum with clean surfaces. However, nanomaterials are seeing increasing real world applications and these require characterisation techniques which can be applied in situ to samples at atmospheric pressure, or even submerged in liquids. SERS is a very effective chemical probe which can meet many of these challenges; it is rapid, inexpensive and gives very detailed structural information on surface chemical structure. Of course, it is limited to nanostructured materials which support appropriate plasmon resonances but these are a significant subset of nanomaterials under investigation.

Received: ((will be filled in by the editorial staff))

Revised: ((will be filled in by the editorial staff)) Published online: ((will be filled in by the editorial staff)) 


\section{WILEY-VCH}

\section{References}

[1] E. Le Ru and P. Etchegoin, Principles of Surface-Enhanced Raman Spectroscopy and Related Plasmonic Effects, Elsevier, Amsterdam, Netherlands, 2008.

[2] S. Schlücker, Surface Enhanced Raman Spectroscopy: Analytical, Biophysical and Life Science Applications, Wiley VCH, Weinheim, Germany, 2010.

[3] S. E. J. Bell, N. M. S. Sirimuthu,, Chem. Soc. Rev., 2008, 37, 1012.

[4] S. Z. Oo, R. Y. Chen, S. Siitonen, V. Kontturi, D. A. Eustace, J. Tuominen, S. Aikio, M. D. B. Charlton, Optics Express, 2013, 21, 18484.

[5] O. Lyandres, N. C. Shah, C. R. Yonzon, J. T. Walsh Jr., M. R. Glucksberg and R. P. Van Duyne, Anal. Chem., 2005, 77, 6134.

[6] S. Mabbott, I. A. Larmour, V. Vishnyakov, Y. Xu, D. Graham, R. Goodacre, Analyst, 2012, 137, 279.

[7] I. A. Larmour, S. E. J. Bell, G. C. Saunders, Angew. Chem. Int. Ed., 2007, 46, 1710.

[8] M. Baalousha, J. Lead, Characterization of Nanomaterials in Complex Environmental and Biological Media, Elsevier, Amsterdam, Netherlands, 2015.

[9] G. Sharma, T. Deckert-Gaudig, V. Deckert, Adv Drug Deliv. Rev., 2015, 89, 42.

[10] J. C. Love, L. A. Estroff, J. K. Kriebel, R.G. Nuzzo, G. M. Whitesides, Chem. Rev., 2005, 105, 1103.

[11] J. Kudelski, J. Raman Spectrosc. 2003, 34, 853.

[12] C. Ma, J. M. Harris, Langmuir, 2012, 28, 2628.

[13] A. G. Brolo, D. E. Irish, B. D. Smith, J. Mol. Struct. 1997, 405, 29.

[14] Y. Wang, A. B. Serrano, K. Sentosun, S. Bals, L. M. Liz-Marzan, Small, 2015, 11, 4314

[15] S. E. J. Bell, N. M. S. Sirimuthu, J. Phys. Chem., 2005, 109, 7405.

[16] C. H. Moran, X. Xia, Y. Xia, Phys. Chem. Chem. Phys., 2013, 15, 5400. 


\section{WILEY-VCH}

[17] A. Stewart, S. Murray, S. E. J. Bell, Analyst, 2015, 140, 2988.

[18] S. E. J. Bell, J. N. Mackle, N. M. S. Sirimuthu, Analyst, 2005, 130, 545.

[19] S. E. J. Bell, N. M. S. Sirimuthu, J. Am. Chem. Soc., 2006, 128, 5580.

[20] W. W. Y. Lee, V. A. D. Silverson, C. P. McCoy, R. F. Donnelly, S. E. J. Bell, Anal. Chem., 2014, 86, 8106.

[21] W. W. Y. Lee, V. A. D. Silverson, Y. C. Ho, L. E. Jones. N. C. Fletcher, M. McNaul, K. L. Peters, S. J. Speers, S. E. J. Bell, Chem. Commun., 2016, DOI: 10.1039/C5CC06745F.

[22] D. Yogev, S. Efrima, J. Phys. Chem., 1988, 92, 5754.

[23] K. C. Gordon, J. J. McGarvey, K. P Taylor, J. Phys. Chem., 1989, 93, 6814.

[24] M. P. Konrad, A. P. Doherty, S. E. J. Bell, Anal. Chem., 2013, 85, 6783.

[25] I. A. Larmour, D. Graham, Analyst, 2011, 136, 3831.

[26] C. Shen, J. Lin, Langmuir, 2012, 28, 640.

[27] A. Stewart, S. Zheng, M. R. McCourt, S. E. J. Bell, ACS Nano, 2012, 6, 3718.

[28] A. Stewart, S. E. J. Bell, Chem. Commun., 2011, 47, 4523.

[29] W. Lin, F Shen. B, Stephanadis, R. Zenobi, Anal. Bioanal. Chem., 2015, 407, 8197.

[30] Y. Cesbron, C. P. Shaw, J. P. Birchall, P. Free, R. Levy, Small, 2012, 8, 3714.

[31] J. Xu, S. Luo, G. Liu, Spect. Acta A, 2015, 143, 35.

[32] D. Li, L. Qu, K. Hu, Y. Long, H. Tian, Angew. Chemie, 2015, 54, 12758.

[33] K. Zhang, J. Zhao, J. Ji, Y. Li, B. Liu, Anal. Chem., 2015, 87, 8702.

[34] K. S. Joya, X. Sala, Phys. Chem. Chem. Phys., 2015, 17, 21094.

[35] X. Y. Ling, R. Yan, S. Lo, D. T. Hoang, C. Liu, M. A. Fardy, S. B. Khan, A. M. Asiri, S. M. Bawaked, P. Yang, Nano Res., 2014, 7, 132.

[36] W. Xie, B. Walkenfort, S. Schlücker, J. Am. Chem. Soc., 2013, 135, 1657.

[37] O. Ivashenko, J. T. van Herpt, B. L. Feringa, P. Rudolf, W. R. Browne, Langmuir, 2013, 29, 4290. 


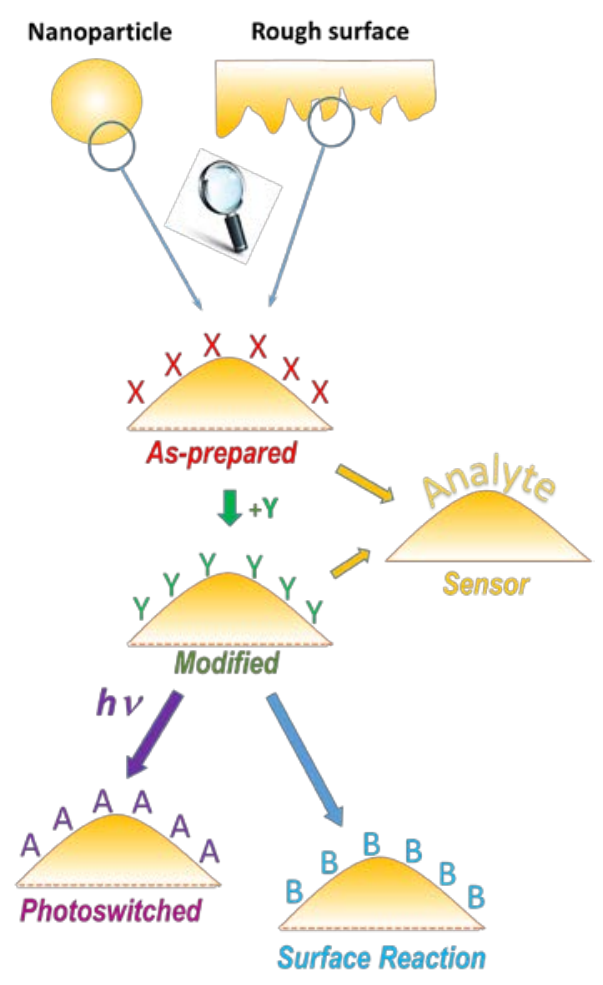

Figure 1. An illustration of the ways that the surface chemistry of SERS active nanomaterials may be changed through chemical or physical processes. The most common use of SERSactive materials is as sensors which interact with analytes to give signals characteristic of the adsorbed target molecule. However, SERS is equally effective for probing chemical modification and reactions on the surface, including photoswitching and catalytic processes. 


\section{WILEY-VCH}
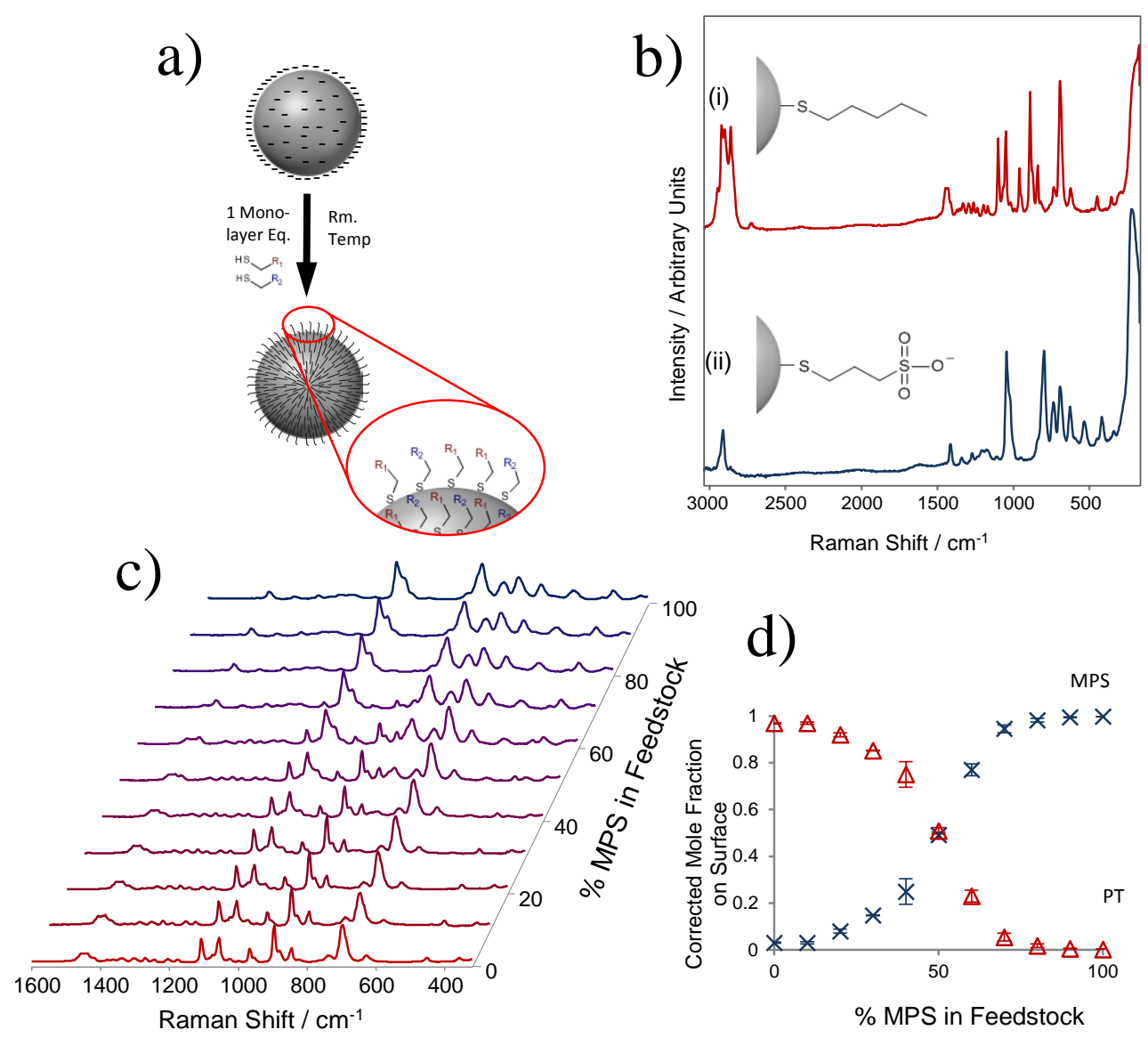

)

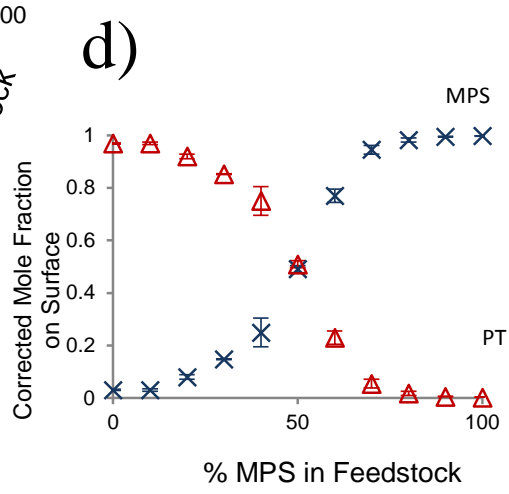

Figure 2. a) Illustration of the modification of a pre-prepared silver nanoparticle with a mixed thiol feedstock. b) SERS spectra of (i) pentanethiol, PT and (ii) mercaptopropanesufonate, MPS adsorbed on Ag colloid. c) SERS spectra of Ag nanoparticles modified using mixed MPS:PT feedstocks with different percentages of MPS. d) Plot of the mole fractions of MPS $(\mathrm{x})$ and PT $(\Delta)$ on the surface obtained at various feedstock compositions. Reproduced with permission. ${ }^{[27]}$ Copyright 2012, ACS Publications. 


\section{WILEY-VCH}

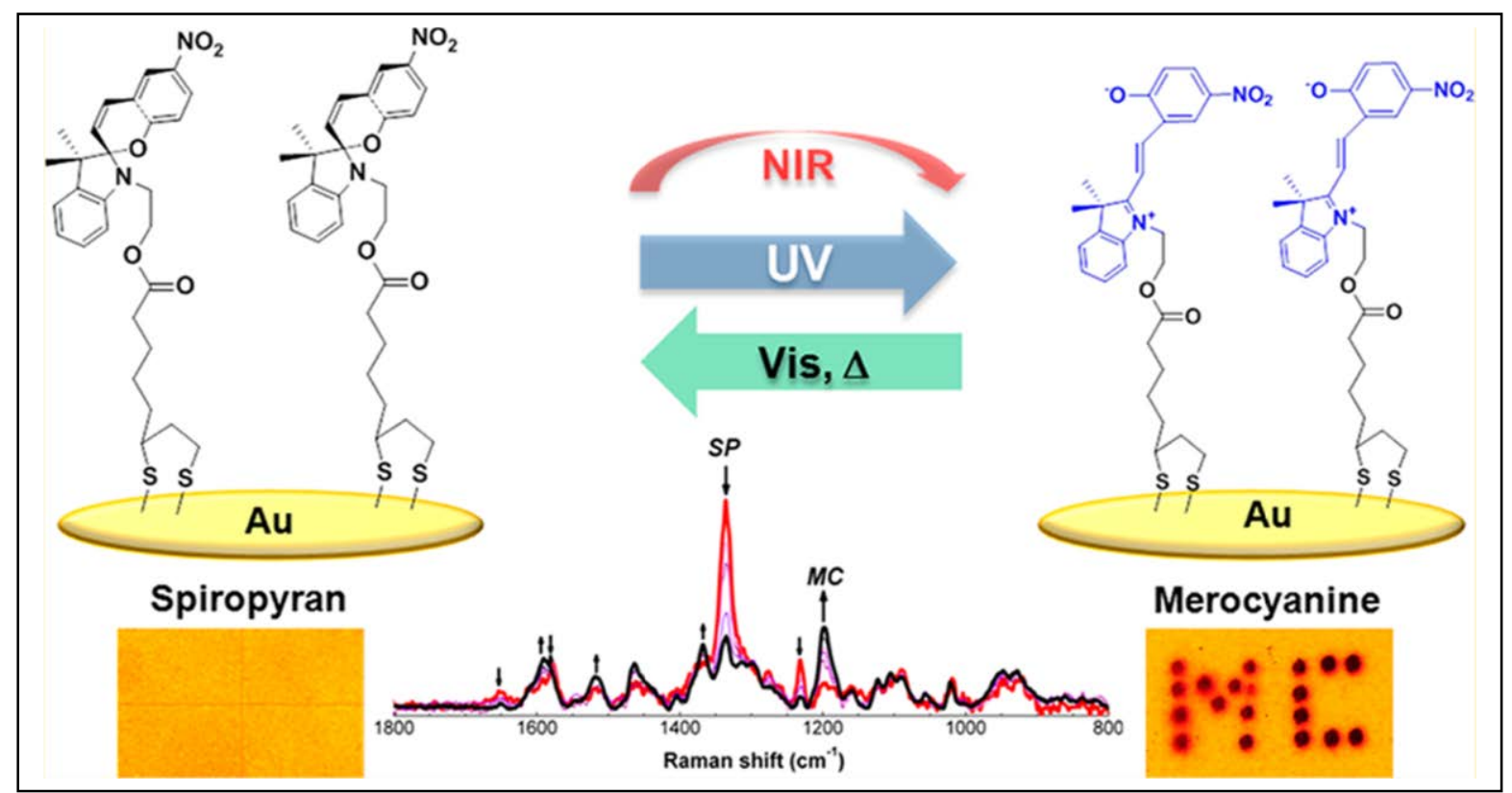

Figure 3. Illustration of switching between ring open (merocyanine, MC) and ring-closed (spiropyran, SP) forms of a spiropyran covalently attached to a Au surface by an alkyl chain and a dithiolate surface anchor. This system can be thermally or optically (785 nm excitation) switched from the MC to the SP form and then reversed using UV excitation. SERS spectra of the ring open and closed forms are shown inset.

Reproduced with permission. ${ }^{[37]}$ Copyright 2013, ACS Publications. 


\section{WILEY-VCH}

Surface-enhanced Raman spectroscopy is a powerful technique for probing the chemical structure of adsorbed surface layers on nanostructured materials. Currently it is predominantly used for detecting adsorption of small molecules but it also provides information on the surface chemistry of enhancing materials themselves. This is important since it is the surface chemistry which governs the interaction of the materials with their environment.

\section{Surfaces}

Susan Dick, Magda P. Konrad, Wendy W. Y. Lee, Hannah McCabe, John N. McCracken, Taifur Rahman, Alan Stewart, Yikai Xu, Steven E. J. Bell*

Surface Enhanced Raman Spectroscopy as a Probe of the Surface Chemistry of Nanostructured Materials

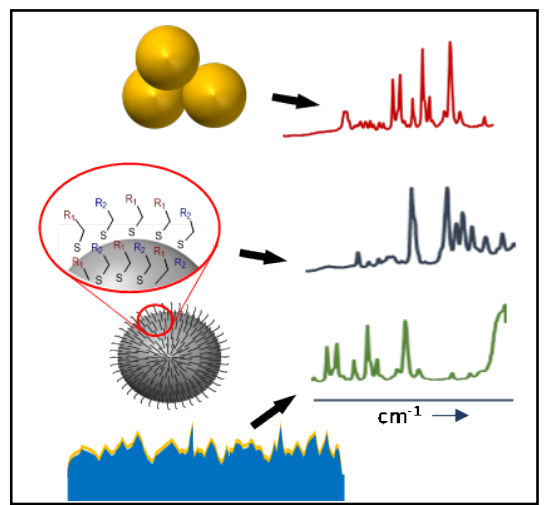

